\title{
Captura desigual de renta de suelo y desplazamiento exclusionario. Indicadores generales del proceso de gentrificación en Santiago de Chile, 2000-2012
}

\author{
Uneven capture of land rent and exclusionary displacement. \\ General indicators of gentrification in Santiago, Chile, 2000-2012 \\ Ernesto López-Morales \\ Ivo Gasic Klet \\ Daniel Meza Corvalán
}

\section{Resumen}

Santiago viene experimentando un proceso de re-estructuración, coherente al ingreso de lógicas neoliberales de producción del espacio desarrollado las últimas décadas. El proceso de renovación urbana del área central y pericentral de la ciudad es en la actualidad el modo de producción espacial dominante, el cual se apropia de espacios bien dotados de infraestructura pública pero que presentan históricamente una baja inversión pública y privada. Se problematiza así la gentrificación, con foco en los procesos de captura desigual de renta del suelo urbano y consecuente desplazamiento exclusionario, en cuatro comunas del área central y pericentral con alta producción inmobiliaria: Recoleta, Ñuñoa, Macul y Santiago.

Palabras clave: Gentrificación; renovación urbana; brecha de renta; desplazamiento.

\begin{abstract}
Santiago is undergoing a restructuring process that is consistent with the neoliberal logic of space production that has been developed in recent decades. The urban renewal process in the city's center and peri-center is now the dominant mode of space production, which appropriates spaces that are well-equipped with public infrastructure but which have historically received low investments, both public and private. The article problematizes gentrification, focusing on the uneven capture of processes of urban land rent and, consequently, on exclusionary displacement, resulting in four districts in the central and pericentral area with high real estate production: Recoleta, Nuñoa, Macul and Santiago.
\end{abstract}

Keywords: gentrification; urban renewal; rent gap; displacement. 


\section{Introducción}

Las ciudades y metrópolis latinoamericanas han experimentado importantes procesos de reestructuración de sus áreas urbanas centrales, consistentes con la adopción generalizada de políticas neoliberales de globalización económica por parte de los Estados del continente en las últimas décadas. Gobiernos nacionales, metropolitanos y locales han promovido transformaciones, orientando progresivamente sus instrumentos para aumentar el potencial de retorno económico para la inversión financiera global y local en mercados inmobiliarios (Smith, 2002). El modelo de administración urbana pro-empresarial, ${ }^{1}$ operando en asociación con productores inmobiliarios, a la par de remodelaciones de la misma estructura estatal para parecerse a la estructura del sector privado (Weber, 2002), encuentra su correlato en Latinoamérica, siguiendo una tendencia global que parece trascender bloques políticos, desde aquellos de corte neoliberal hasta, paradojalmente, los más progresistas (Hackworth, 2007). En cuanto a las estrategias, si bien han sido múltiples, generalmente pasan por asociaciones e instrumentos de promoción, que las administraciones locales municipales urbanas utilizan como medios para consolidar la expansión de una lógica de mercantilización del espacio urbano (Rojas, 2004; Herzer, 2008; Delgadillo, 2008; López-Morales, 2011; Janoschka, Sequera y Salinas, 2013).

En Chile, con el objetivo de recuperar espacios de alta centralidad y revertir los efectos de al menos tres décadas previas de despoblamiento por migración centrífuga hacia la periferia, y descenso del nivel socioeconómico promedio de los hogares residentes centrales, el Estado desde mediados de la década de 1980 comenzó un proceso de configuración de líneas estratégicas públicas y municipales de renovación urbana a materializarse por agentes privados, a saber: a) implementación de agencias privadomunicipales para atraer y anclar al centro y pericentro el desarrollo inmobiliario; b) aplicación de un subsidio para la adquisición de viviendas económicas (inferiores a $140 \mathrm{~m}^{2}$ ) en un área definida como Zona de Renovación Urbana; y c) orientación de los Instrumentos de Planificación Territorial (IPT) para establecer condiciones que posibiliten el desarrollo inmobiliario en las diferentes coyunturas, redistribuyendo complementariamente otros usos del suelo funcional a la densificación habitacional. Específicamente, lo último implica la disposición de normativas de regulación municipal del suelo a fin de mejorar la competitividad urbana local para la atracción de capital inmobiliario privado; la tendencia a la institucionalización de espacios decisionales público-privados, que expresan un poder no monopólico sino competitivo (Gajst, 2010) sobre la toma de decisiones en asuntos de gestión, habilitación de suelos y producción inmobiliaria; la absorción, por parte de los gobiernos locales, de los riesgos políticos que genera el proceso de renovación ante la ausencia del gobierno central como ente activo; y la fragmentación de la planificación racional-holística del Estado con reemplazo de lógicas de gestión y planificación público-privada más flexibles y compartimentadas (López-Morales, Gasic y Meza, 2012; Vicuña, 2013). 
En la actualidad la renovación de zonas centrales tiene un alcance nacional. Inicialmente Santiago, en su zona central ha sido el lugar de origen y maduración de un modelo importado a otras ciudades. Coherente con una importante centralización política, la comuna de Santiago y alrededores es el espacio donde se ponen en práctica varias estrategias articulación para la renovación central con resultados a largo plazo. Se estima que desde 2007 en adelante, el volumen construido y de unidades habitacionales producidas por el modelo privado de renovación urbana en altura en comunas centrales y pericentrales del Área Metropolitana del Gran Santiago (AMGS) es mayor que el de la construcción de casas unifamiliares en la periferia. Asimismo, la participación de la zona central y pericentral alcanza un $44 \%$ de la producción de vivienda. Dichas acciones de renovación urbana han revertido la lógica actualmente imperante de localización del desarrollo urbano en Santiago de Chile. La ciudad es, en rigor, una metrópolis que se verticaliza.

De esta forma, diversos espacios son intervenidos por un proceso de reemplazo de material construido. Se genera así un escenario donde un proceso tiene diversas facetas. Las particularidades en cada comuna devienen por un lado de las disposiciones regulatorias que cada comuna establece para sus diferentes espacios (cada una de las 34 comunas que compone el Gran Santiago elabora su instrumento de acuerdo a un plan de desarrollo), y la situación anterior de las zonas que son intervenidas por la renovación.
En ciertos casos, el municipio establece una estrategia pública de ir adaptando los instrumentos regulatorios para cada fase del proceso de renovación urbana en su comuna. En una suerte de distribución de la inversión y cierre de zonas saturadas, guiando así el proceso dentro del territorio. En otros, la condición anterior de espacios con baja inversión pública y privada en los inmuebles, hacinamiento en construcciones antiguas (buena parte de ella población inmigrante), subutilización y falta voluntad para invertir por parte de los propietarios, generan diversos escenarios de relaciones sociales y mercantilización del suelo.

La fase de intensa producción inmobiliaria que se ha desplegado durante la década de los 2000 en estas zona provocando emigración de residentes tradicionales, mediante proyectos inmobiliarios que compran, en promedio, una serie de 4-5 terrenos para edificar. Se vuelve relevante la pregunta acerca del grado de acceso al mercado habitacional in situ que tienen los residentes actuales en las zonas en desarrollo, para estimar posibilidades de relocalización y problematizar las situaciones de expulsión o desplazamiento de los mismos hacia la periferia urbana. Probablemente la pregunta central acerca de todo este proceso sea: ¿Hay o ha habido gentrificación en el pericentro de Santiago de Chile, dada su condición estructural de ser una zona atravesada por un proceso de renovación urbana intensificado y por albergar población residente de estratos medio, medio-bajos y bajos? 
El presente artículo aborda el estudio de cuatro zonas de concentración inmobiliaria de la zona centro y pericentro del Gran Santiago: Recoleta (comuna de Recoleta), Irarrázaval (comuna de Ñuñoa), Macul (comuna de Macul) y Santa Isabel (comuna de Santiago), en las que se realiza una aproximación cuantitativa del fenómeno. Se delimitaron polígonos de estudio en estas zonas de concentración del mercado inmobiliario, como se muestran en la Figura 1, a partir de lo cual se analizaron los proyectos desarrollados durante el periodo 2000-2012.

Figura 1 - Mapa de las zonas de estudio
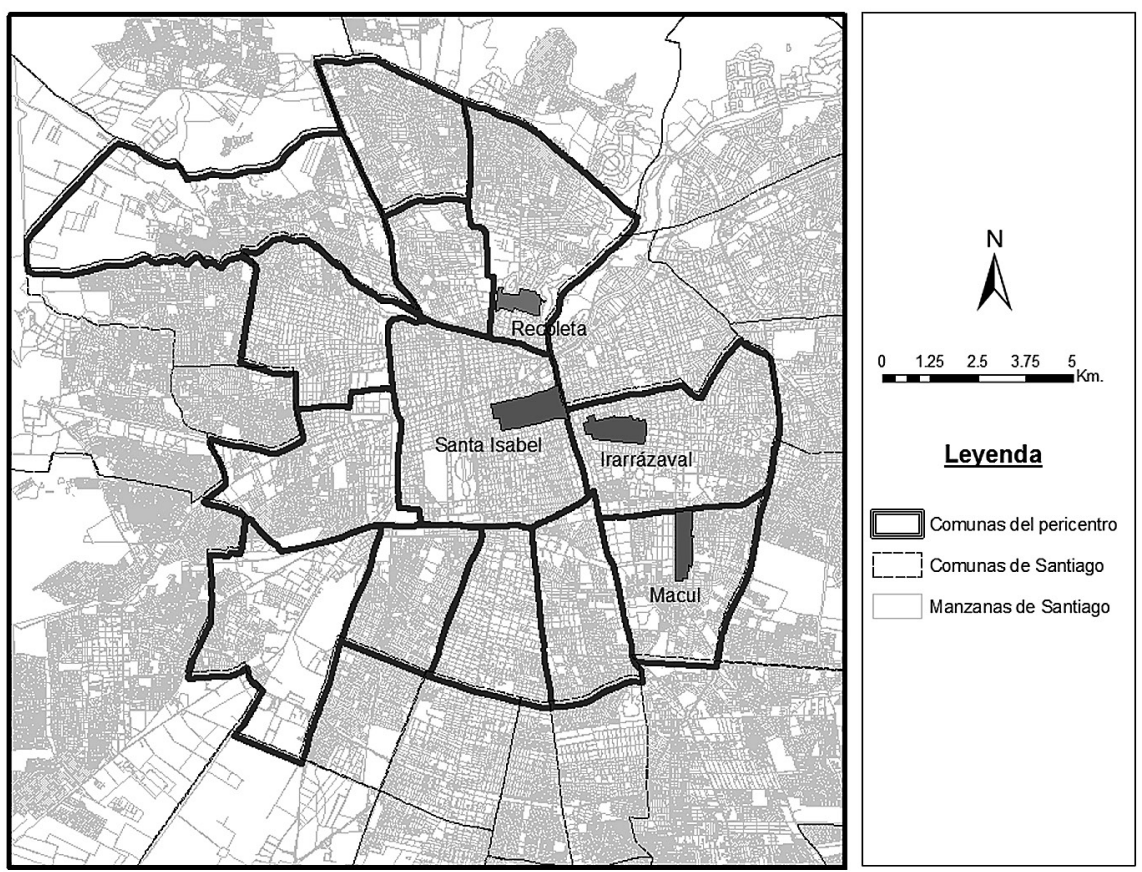

Fuente: Elaboración propia. 


\section{Construyendo un marco teórico crítico para abordar el problema de la gentrificación en Santiago de Chile}

De partida, el concepto de gentrificación está atravesado por importantes discusiones y disputas académicas, ideológicas e incluso políticas (Slater 2006, 2009; Shaw, 2008; Janoschka y Casgrain, 2011). Sin embargo, por lo pronto, es posible distinguir enfoques culturalistas o centrados en la demanda y enfoques estructuralistas, de la oferta 0 de la producción espacial (Lees, Slater y Wyly, 2008). Por una parte, el enfoque cultural basa su interpretación del fenómeno en la emergencia de nuevas preferencias locacionales de la vivienda en algunas ciudades europeas principalmente Londres - y norteamericanas. Aduce a cambios culturales y sociodemográficos que re-posicionan la demanda residencial por el suelo urbano central, generando nuevas inversiones inmobiliarias en barrios centrales deteriorados (Herzer, 2008; Lees, Slater y Wyly, 2008). La tendencia a la reducción de los integrantes de los núcleos familiares, como la emergencia de nuevas clases medias, son algunos factores sociodemográficos que estarían incidiendo en el aumento de la demanda por suelo central (Herzer, 2008). Las tendencias culturales de grupos alternativos como artistas, bohemios y otros, también son consideradas como factores culturales que conducen a la renovación y gentrificación de un barrio.

En cuanto al enfoque estructural, éste basa su interpretación en la emergencia de nuevas posibilidades para la realización del capital inmobiliario en las zonas centrales (Herzer, 2008). Más allá de las nuevas tendencias culturales y socio-demográficas, el factor que condiciona el proceso es la disponibilidad de capitales y su capacidad para movilizarse y producir espacio. Desde este enfoque, los gentrificadores no son necesariamente los grupos sociales que llegan a vivir a un barrio, sino la clase social que genera producción inmobiliaria a mayor escala y que estructura la oferta y el desarrollo posterior del barrio (López-Morales, 2008, 2010). Al poner el centro del análisis en las relaciones de clase, se quiere relevar el hecho de que en la producción del espacio existen procesos de apropiación material del trabajo socialmente realizado, en forma de renta de suelo urbano. De acuerdo a esta definición, la gentrificación debe ser estudiada como proceso de des-y re-inversión de capital en zonas urbanas consolidadas, generando mecanismos de des- y re-poblamiento con nuevos patrones espaciales de composición socio-económica de la población. En rigor, la gentrificación desde el enfoque estructural puede resumirse en el imperativo título del primer artículo de Neil Smith (1979) sobre la cuestión: una vuelta a la ciudad movilizada por el capital, no por las personas.

Para el caso de esta investigación se utiliza el enfoque de la producción espacial, con el objeto de observar relaciones de clase en la re-estructuración capitalista del espacio urbano central. Se entiende la gentrificación, entonces, como un proceso de re-mercantilización del suelo urbano central con efectos de exclusión social, particularmente desplazamiento socioresidencial de habitantes originarios (Smith, 1996, 1979; Marcuse, 1985; Clark, 1987; 
Hackworth, 2007; López-Morales, 2008, 2009, 2010). Esto implica, al menos, comprender tres estadios o eventos procesuales: 1) producción y regulación de renta en suelos urbanos centrales; 2) apropiación privada de renta urbana por actividad inmobiliaria intensiva; 3) proceso de desplazamiento y exclusión de los residentes originarios que no pueden costear el arrendamiento o la compra de las nuevas viviendas. Para el propósito de nuclear estos aspectos en un marco teórico, se revisa la Teoría de la Brecha de Renta de Neil Smith (1979, 1996), considerada como un aporte de carácter conceptual-crítico que debe servir de orientación heurística para investigaciones empíricas.

La teoría del rent gap (traducida al español como brecha de renta por López, 2008), propuesta originariamente por Neil Smith (1979) para entender los procesos de gentrificación en ciudades norteamericanas, concibe la renovación urbana como un proceso de producción espacial que detona reinversión inmobiliaria; siendo ésta un modo de apropiación de la renta posible de extraer de suelos centrales y peri-centrales. La reinversión inmobiliaria surge, precisamente, cuando se combina el efecto de depreciación del suelo en su situación actual de uso y el efecto de apreciación del suelo por su condición estructural de localización. ${ }^{2}$ Ahora bien, esta teoría requiere de una aplicación en Santiago de Chile coherente a la estructura de clases sociales que componen las áreas centrales. Esto quiere decir: emplear las herramientas conceptuales formuladas por Smith (1979, 1996) para analizar las relaciones existentes entre las clases sociales propietarias de los factores productivos - suelo, capital, trabajo - que producen la renovación urbana. Para estos efectos deben considerarse las siguientes características de las áreas urbanas centrales de Santiago de Chile:

1) Concentración del suelo urbano: el modelo presentado por Smith (1979, 1996), y utilizado para analizar procesos de gentrificación en ciudades del "Norte Global" (clásicamente ciudades del sunbelt norteamericano, Canada, Europa occidental y Australia), se basa en estructuras de alta concentración de la propiedad del suelo, donde existen grandes rentistas de suelo urbano que alquilan vivienda. En contraste, las políticas de vivienda aplicadas desde los años 60' en Chile han consistido en la entrega de suelo central a residentes de segmentos medios y medio-bajos; lo que trae por consecuencia una actual tasa de propietarios-residentes en las zonas de renovación del centro de Santiago cercana al 80\% (López-Morales, 2013). El modelo chileno de propiedad privada del suelo urbano para los residentes implica, por ende, una estructura de propiedad desconcentrada, donde prima el valor de uso del suelo por sobre la extracción de renta de alquiler, pero donde también la propiedad minúscula de suelo se configura como el principal patrimonio para una gran mayoría de hogares urbanos. En rigor, persisten pequeños rentistas que históricamente han alquilado sus viviendas a segmentos populares, incluso actualmente aprovechando la alta demanda habitacional por inmigrantes latinoamericanos (Borsdorf e Hidalgo, 2013), pero que no poseen el volumen de suelo ni de capital para poder materializar el volumen de edificación suficiente para absorber toda la renta potencial existente en el lugar. 
2) Concentración del capital inmobiliario: otra diferencia estructural respecto a las ciudades del Norte Global es la alta concentración del capital inmobiliario que detona la renovación urbana en Santiago. Mientras en el modelo de Smith $(1979,1996)$ quien detona la inversión inmobiliaria es la clase rentista, poseedora de suelo y cierto capital inmobiliario, en el caso de Santiago la alta corporatización y concentración de capitales inmobiliarios acelerada por las políticas neoliberales aplicadas desde mitad de la década del 70' (De Mattos, 2006, 2007) configura un escenario de exploración inmobiliaria por las áreas centrales en búsqueda de suelos para renovación. Sobre todo si se considera que desde entonces se viene produciendo una integración productiva de la actividad constructiva con la gestión inmobiliaria privada, a través de lo que denominara Sabatini (1990) como Sistema de Promoción Inmobiliario (SPI). Además de la concentración de agentes productivos y la integración de la actividad constructiva en sistemas de promoción más complejos, Cattaneo (2011) identifica que las reformas al mercado de capitales han implicado la participación nuevos agentes financieros, holdings, y fondos de inversión privados con preferencia para localizar sus proyectos inmobiliarios en áreas centrales.

3) Producción de renta: las reflexiones provenientes del Norte Global centran la atención en la escala local de producción de renta (Smith, 1996), basada en las restauraciones y revitalizaciones de las áreas centrales impulsadas por una pequeña-burguesía ${ }^{3}$ compuesta de yuppies, jóvenes artistas, bohemios, etc. Si bien la relación entre lo local y metropolitano es parte de una dialéctica de producción social de escalas espaciales, donde se producen constantes re-escalamientos, es posible señalar que en Santiago de Chile el proceso de renovación urbana ha consistido predominantemente en la producción de renta de suelo a escala metropolitana (López-Morales, 2010a). Las iniciativas pioneras de revitalización de las áreas centrales no parecieran ser generativas del impetuoso proceso de renovación urbana comandado por grandes corporaciones de capital inmobiliario. Los casos del Barrio Lastarria, Barrio Bellavista, Barrio Italia ${ }^{4}$ y otros, constituyen tal vez primeras fases de procesos de gentrificación, pero en la actualidad no es sensato indicar que sean estas iniciativas las que generan las condiciones económicas para la renovación urbana.

Sintetizando los elementos planteados, la diferencia sustancial del proceso de gentrificación en Santiago respecto al Norte Global consiste en que la confrontación económica principal se realiza entre un capital inmobiliario altamente corporatizado y pequeños propietarios-residentes. La figura de los revitalizadores no parece sustantiva en la producción de renta potencial, lo que permite sostener que: aun cuando pueda realizarse en algunos barrios históricos del centro de Santiago, con características empíricocontingentes similares a casos de ciudades europeas (Schlack y Turnbull, 2011; Inzulza-Contardo, 2012), la colonización de estos grupos pioneros no ha reportado atracción para el capital inmobiliario de renovación a gran escala. Mientras Hackworth (2007) señala que en ciudades del Norte Global este tipo de colonización pionera corresponde a una etapa inicial del proceso de gentrificación, que luego 
se corporatiza por capitales inmobiliarios externos al barrio (escala local), en Santiago no pareciera generarse dicho desarrollo. Pareciera que la alta concentración de estos capitales en Chile y su fácil acceso a suelo central a través de fusión de predios de pequeños propietarios produce condiciones suficientes para una producción capitalista concentrada.

Como ha venido avanzando López (2009, 2010a, 2010b), el centro del debate de la gentrificación en Chile debe girar fundamentalmente en torno a las relaciones de confrontación económica entre la clase inmobiliaria y los pequeños propietarios residentes. Más aún cuando la nula regulación estatal en los precios y la cantidad de vivienda ofertada, y la integración de la actividad constructiva en sistemas vinculados a la gestión y promoción inmobiliaria, generan un escenario donde las ganancias de los agentes inmobiliarios se componen fundamentalmente de las rentas del suelo, más que de la mejora técnica en la construcción de la vivienda (Sabatini, 2000). Lo que se deriva, hipotéticamente, es una fuerte disputa por determinados suelos en las áreas centrales de alta renta para la actividad inmobiliaria; generando presión a la venta de predios por parte de los propietarios-residentes. Si bien los propietarios del suelo se pueden ver beneficiados a medida que aumenta la demanda de los agentes inmobiliarios, elevando los precios del mismo (Sabatini, 2000), lo cierto es que esto depende del impacto que el mismo proceso de renovación va generando en la zona.

Ahora bien, tras la producción material del espacio urbano y la apropiación privada de renta, es necesario completar la totalidad del concepto de gentrificación conceptualizando de manera operativa las situaciones de expulsión, exclusión, desplazamiento, desposesión, etc. Para esto se propone el concepto de desplazamiento exclusionario legado por Marcuse (1985), haciéndolo operativo al caso de la renovación urbana intensiva en Santiago. Se define, entonces, como la imposibilidad o dificultad de relocalización de los propietarios-residentes en las viviendas ofertadas en la misma zona en la que han vendido sus predios. Esto implica que la re-mercantilización del suelo urbano central, que compele a los residentes a comportarse como oferentes, deviene no solo en apropiación desigual de renta sino en desplazamiento e imposibilidad de relocalización de los residentes en las mismas áreas de actividad inmobiliaria de renovación. Si bien se reconoce como un proceso complejo y de múltiples determinaciones, este desplazamiento exclusionario puede medirse en función de tasas de relocalización; entendidas como la relación entre la renta obtenida por los propietarios-residentes por la venta de sus predios y el precio de las viviendas requeridas para relocalización en la misma zona.

Considerando esta estructura teórica, se sostiene hipotéticamente que la gentrificación en Santiago es un proceso de remercantilización del suelo urbano central por ingreso de capital inmobiliario de renovación urbana que comporta apropiación desigual de renta urbana con efecto de desplazamiento exclusionario de los residentes originales de las áreas centrales. En base a esta hipótesis se orienta un trabajo de campo con recolección y análisis de datos cualitativos y cuantitativos en la zona de Santa Isabel, comuna de Santiago. 


\section{Metodología}

Para caracterizar el proceso de gentrificación por desposesión, en su modo particular de producción espacial, se planteó un trabajo de análisis cuantitativo, en zonas específicas de concentración inmobiliaria y promoción estatal de la renovación urbana, orientado en torno a tres aspectos claves: a) estimación de la renta de suelo capitalizada por desarrolladores en cada proyecto de renovación urbana, b) cálculo de la renta de suelo de propietarios anteriores (e intermediarios), y c) proyección de una tasa de relocalización.

El caso de estudio del pericentro de Santiago, abordado en el presente artículo, es aplicado a seis polígonos de concentración de proyectos residenciales en altura desarrollados durante el periodo 2000-2012. Se analiza un total de 163 proyectos, para los cuales se realizó una revisión de expedientes en el archivo de la Dirección de Obras Municipales de Santiago (DOM), develando las características generales de arquitectura, volumen de la construcción y tipologías de departamentos ofertados al detalle. Estas últimas fueron vinculadas a la revisión de precios de la oferta inmobiliaria en plataformas digitales especializadas (portal inmobiliario.com), para cada proyecto y su correspondiente tipología específica. Sistematizando estos insumos en una tabla de correlaciones (Figura 2) se estima un valor final correspondiente a la renta de suelo capitalizada en cada proyecto por concepto de venta de departamentos, restando costos de producción, derechos municipales (impuesto público a la construcción) y costos blandos (publicidad y difusión), sin contar el gasto por el suelo desarrollado. Este valor es denominado RCS-2 (Renta Capitalizada de Suelo 2), el cual también es expresado en términos absolutos (divido por la superficie del terreno desarrollado) para establecer comparaciones y develar tendencias.

Paralelamente se indagó en los registros del Conservador de Bienes Raíces de Santiago (CBRS), generando un historial de los predios comprados para cada proyecto. Concretamente en el caso del pericentro del Gran Santiago, se levantó un total de 568 transacciones de propiedades anteriores, de las cuales 417 corresponden a propiedades con uso efectivo anterior (propietarios con inscripción anterior previa al inicio del proceso de renovación urbana, 1990) y 151 propietarios intermedios que denominamos intermediarios. Como muestra la Figura 3, el valor final generado corresponde al promedio de la renta obtenida por propietarios anteriores (Renta de Suelo Capitalizada 1 o RCS-1).

A partir de estos dos valores (RCS1 y RCS-2) se calcula una brecha de renta, estimada como la ganancia final obtenida por el productor inmobiliario, por unidad de superficie de suelo, descontando todos los costos asociados a la ejecución del proyecto. En clave de fórmula: $\mathrm{Br}=[\mathrm{RCS}-2-\mathrm{RCS}$ 1] / Sup, donde $S$ es la superficie del predio desarrollado, en metros cuadrados. Se plantean dos supuestos para el cálculo: que todas las unidades son vendidas por proyecto, y se estima su rentabilidad en un periodo anual sin cálculo de Valor Actualizado Neto (VAN) para años sucesivos. Ambos supuestos son posibles en un mercado con velocidades de venta generalmente altas. En cualquier caso, se trata de un cálculo de brecha de renta tendencial 
Figura 2 - Ejemplo de Ficha Inmobiliaria. Identifica proyecto, dirección del predio, características arquitectónicas, características de la oferta y costos (1UF=46US $\$=100 R \$)$
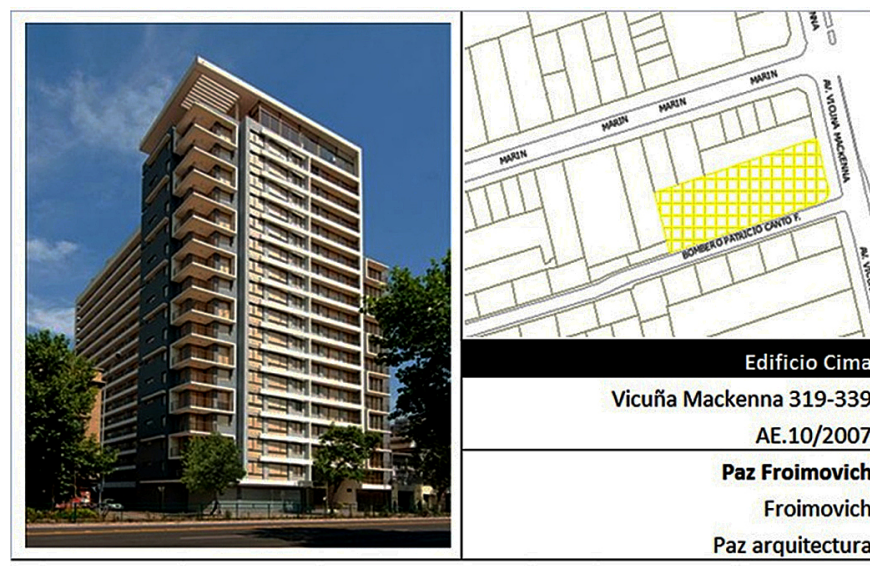

\begin{tabular}{|c|c|c|c|c|}
\hline \begin{tabular}{l|l} 
& Sup. predial final $\left(\mathrm{m}^{2}\right)$ \\
Ocupación de suelo $\left(\mathrm{m}^{2}\right)$ \\
Edif. sobre NTN $\left(\mathrm{m}^{2}\right)$
\end{tabular} & $\begin{array}{r}2.014 \\
544 \\
16.234 \\
3.816 \\
1.470 \\
\end{array}$ & $\begin{array}{l}\text { Coef.Constructibilidad } \\
\text { Coef.Ocupación de suelo } \\
\text { Altura de la Edificación } \\
\text { Zona PRS } 1989 \\
\text { Sist. de Agrupamiento }\end{array}$ & $\begin{array}{r}6,52 \\
0,27 \\
18 \text { pisos } \\
\mathrm{Z} \mathrm{B} \\
\text { A sobre C }\end{array}$ & $\begin{array}{r}\text { Características del volumen } \\
\text { general proyectado }\end{array}$ \\
\hline
\end{tabular}

\begin{tabular}{|l|r|r|r|r|r|r}
\hline Tipologías & UF/m2 & $\mathrm{m} 2$ & UF & cantidad & \\
\hline $1 \mathrm{D}-1 \mathrm{~B}$ & 50,37 & 23,05 & 1161 & 24 & \\
& 50,37 & 28,19 & 1420 & 119 & \\
& 50,37 & 28,73 & 1447 & 38 & \\
& 50,37 & 30,29 & 1526 & 18 & \\
& 49,87 & 31,08 & 1550 & 22 & \\
\\
\end{tabular}

Fuente: Elaboración propia con información de la DOM de Santiago y portalinmobiliario.com. 
Figura 3 - Ejemplo de Ficha de Transacción de suelo. Identifica proyecto, dirección del predio, superficie y precio (1UF=46US\$=100R \$)

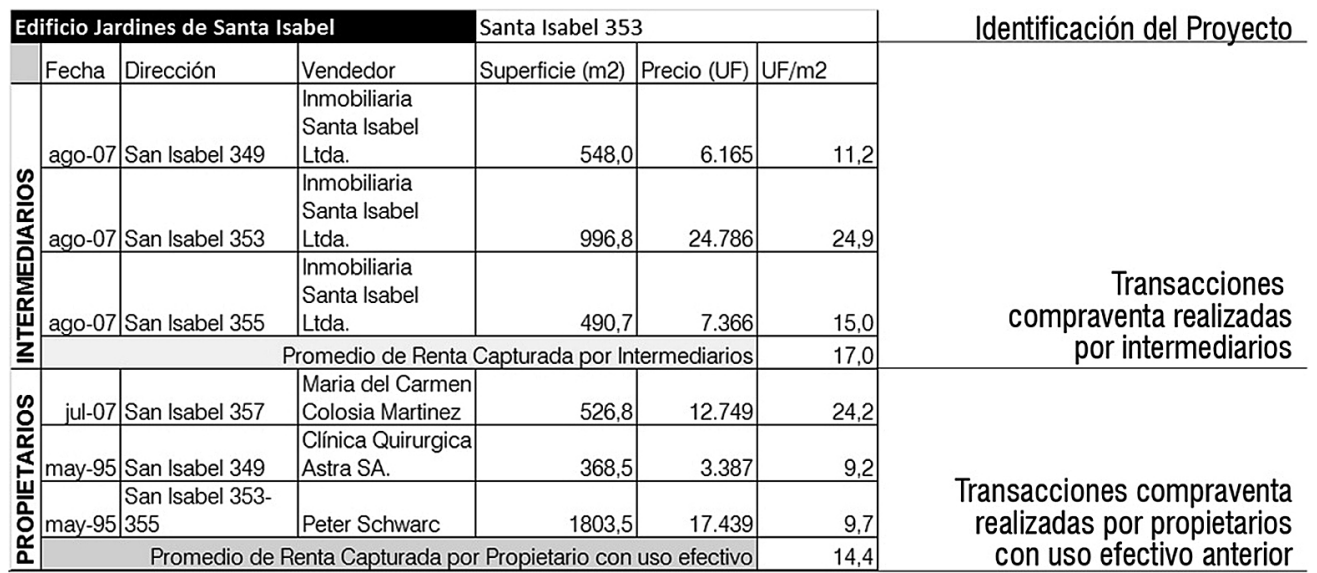

Fuente: Elaboración propia con información del Conservador de Bienes Raíces de Santiago.

y con fines comparativos, con una muestra considerable de proyectos.

Se estima además una "tasa de acumulación", referida al mismo diferencial de la brecha de renta definida como la relación de división entre la brecha de renta capturada por cada proyecto inmobiliario y la renta capturada por los propietarios anteriores: $\mathrm{Ta}=[\mathrm{RCS}-2$ / RCS-1].

Finalmente se calcula una tasa de relocalización para actuales propietarioresidentes de las zonas de renovación. A partir del levantamiento de una encuesta a vecinos de las zonas de renovación, ${ }^{5}$ se simula la renta de suelo que obtendrían actuales propietarios-residentes, a fin de ver las posibilidades de relocalización en proyectos de nueva construcción por renovación urbana. Para ello se relaciona dicha renta simulada con el precio de oferta de la tipología que le correspondería según el número de personas que compone el hogar. La Tasa de relocalización ( $\mathrm{T}$ ) es una medida que se resume en la fórmula: $\mathrm{Trl}=$ [RCS-1prom * Sup] / Pdepto, cuyos factores son: a) promedio de la renta unitaria obtenida por residentes anteriores (RCS1 prom), Superficie de la propiedad (Sup), y Precio de Departamentos ofertados (Pdepto). Este último factor consideró el precio de la oferta actual de departamentos en la zona según tipología correspondiente a cada caso (dividida en categorías de 1, 2, 3 y 4 dormitorios) ${ }^{6}$ y se establecieron medidas de precio mínimo, máximo y promedio. Se plantea como supuesto el uso del total de la RCS-1 para el pago de una propiedad. 


\section{Resultados}

\section{Ensanchamiento de la brecha de renta del suelo}

Como primer resultado de interés, se puede señalar que el conjunto de las zonas estudiadas comporta un notable ensanchamiento de la brecha de renta de suelo durante el período de 2000 a 2012. Éstas, al final del período, expresan un aumento del $104 \%$ respecto del inicio del mismo. De manera más específica, se aprecia un punto de inflexión en 2007, ya que antes de este año las brechas promediaban menos de $45 \mathrm{UF} / \mathrm{m}^{2}$, y después del mismo se mantienen en un rango entre 50 y $80 \mathrm{UF} / \mathrm{m}^{2}$. Así, los años 2006 y 2007 son los que cuentan con mayor cantidad de registros y permiten hablar de un aumento importante de las brechas de renta en el período de estudio, en extremo comparando 2000 y 2007, donde el registro para este último llega a $72 \mathrm{UF} / \mathrm{m}^{2}$, casi siete veces lo obtenido en el año 2000.

Sin embargo, mientras las utilidades inmobiliarias obtenidas por unidad de suelo desarrollado (brechas de renta) aumentan sostenidamente, las rentas capturadas por los propietarios de los predios (RCS-1) no comportan variación significativa, reportando valores similares durante todos los años del período. A saber, se observa una variación de menos de un 5\% entre 2000-2012, con valores que fluctúan entre las 9 y $13 \mathrm{UF} / \mathrm{m}^{2}$ (Figura 4). Si bien estos valores representan precios de suelo relativamente altos para el mercado de suelo de Santiago, corresponden a una porción menor de las ganancias obtenidas por el

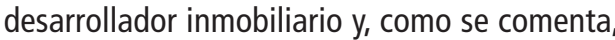
tampoco ascienden en el período estudiado. Esto permite des-mitificar el supuesto de que el precio de éste aumenta ${ }^{7}$ en la medida en que penetra progresivamente la producción inmobiliaria en una determinada zona.

Figura 4 - Evolución de la renta capitalizada de suelo 1 y la brecha de renta.

(1UF=46US $\$=100 R \$$ )

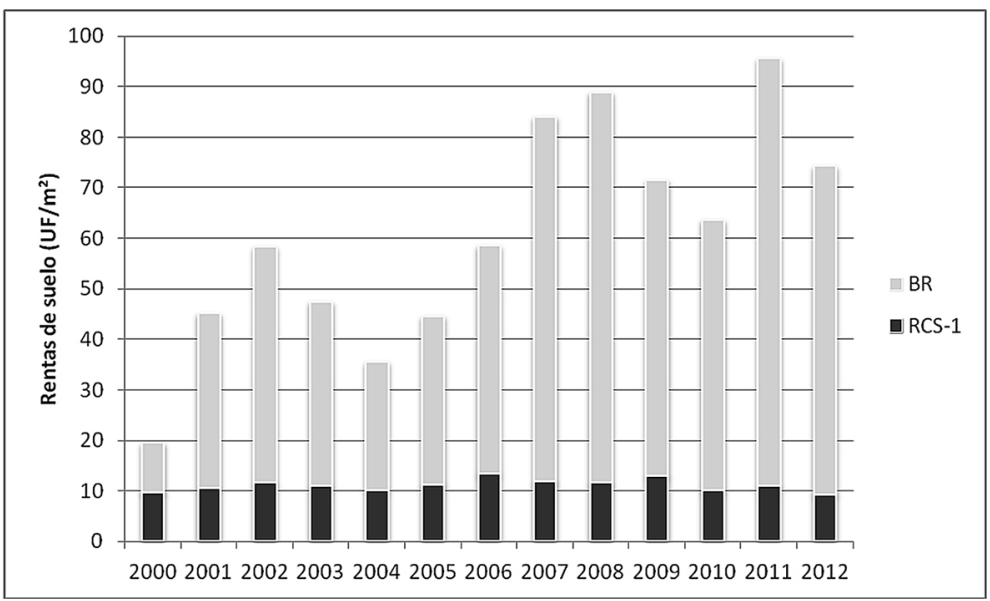

Fuente: Elaboración propia. 
Las razones de este comportamiento pueden ser variadas. Principalmente, debe señalarse que el mercado inmobiliario de renovación urbana está bastante concentrado (López-Morales et al., 2013), sobre todo si se mide relativo a la des-concentración de la propiedad del suelo existente en las zonas estudiadas. A saber, en las cuatro zonas predominan predios relativamente pequeños, en algunas zonas incluso inferiores a $300 \mathrm{~m}^{2}$, donde deben fusionarse cerca de cuatro propiedades para lograr un proyecto inmobiliario. Esta situación, si bien disímil entre las zonas, genera que el desarrollador inmobiliario pueda comprar una propiedad $y$, mediante el cambio en la estructura funcional y de usos de suelo en la zona que impliquen baja en la calidad de vida residencial, pueda negociar con el resto de los propietarios. Por ejemplo, se ha observado que algunas empresas inmobiliarias compran suelo y luego dejan las propiedades sin uso, deteriorando el hábitat residencial y presionando a la venta de las propiedades.

Asimismo, como esta producción inmobiliaria se expande de manera compacta en el espacio urbano, también se van generando externalidades ambientales sobre las unidades residenciales más bajas, producto de la edificación en altura de los proyectos de renovación. en la encuesta más del 50\% de los encuestados perciben efectos de interrupción visual y congestión. Tal como planteara Smith $(1979,1996)$ para referirse al blockbusting (presión inmobiliaria) y al blow-out (estallido de residentes) como mecanismos de expulsión de arrendatarios en áreas de alta renta en ciudades del Norte Global, es posible interpretar el impacto de la renovación urbana intensiva en
Chile como un mecanismo que expulsa a los propietarios-residentes devaluando su hábitat residencial.

Independiente de tratarse de estrategias deliberadas desde el sector inmobiliario, lo cierto es que se ha constatado en las encuestas que la actividad constructiva y las operaciones inmobiliarias asociadas (deterioro intencionado de predios vecinos, especulación de agentes intermediarios, desocupación de inmuebles adquiridos por los productores inmobiliarios, etc.) producen una pérdida en el valor de uso de los residentes, lo cual puede presionar a que las rentas por venta de sus propiedades no tiendan al alza. Si se suma a la concentración de los agentes productores, lo que se obtiene es una situación de mercado monopsónica en donde los propietarios-residentes tienen solo un comprador, que ya se ha instalado en la zona modificando la calidad de vida residencial, y donde es posible prever que tengan escaso poder de negociación.

\section{Brechas de renta y RCS-1 por entidades espaciales}

Al revisar los resultados por entidades espaciales, se obtiene que la zona con mayor RCS-1 es Irarrázaval, con valores de $16 \mathrm{UF} / \mathrm{m}^{2}$. En efecto, en esta zona se ubican los suelos de mayor precio dentro del pericentro que ha acogido procesos de renovación urbana en Santiago de Chile. De ahí, las otras tres zonas presentan valores similares, entre las $10 \mathrm{y}$ $11 \mathrm{UF} / \mathrm{m}^{2}$, significativamente por debajo de Irarrázaval. Esta diferencia radica en que ésta es una zona tradicionalmente de segmentos sociales medios, con ingresos mayores al resto 
de las zonas, y con un deterioro físico del espacio urbano menor. Es una zona en donde las empresas inmobiliarias compran suelo a mayor precio, pero así mismo re-valorizan las nuevas edificaciones en altura con precios de vivienda mayores al resto del pericentro.

En cuanto a las brechas de renta, graficadas en la Figura 5, la zona con valores más altos es Santa Isabel, con cifras que alcanzan las $80,4 \mathrm{UF} / \mathrm{m}^{2}$, lo que supera ampliamente al resto de las zonas estudiadas. Evidentemente que este resultado puede explicarse por la intensidad de producción permitida y realizada en Santa Isabel, con edificaciones que alcanzan los 30 pisos, y con un promedio de 301 departamentos por proyecto. Además, los proyectos en Santa Isabel se desarrollan, en muchos casos, sobre superficies menores a 1.500 $\mathrm{m}^{2}$, erigiéndose como la zona con proyectos de menor superficie predial promedio. Esto, sumado a una alta intensidad de producción y un precio medio de las viviendas ofertadas en relación al resto del pericentro estudiado, la posiciona como la zona con mayor brecha de renta.

Figura 5 - Comparación por zona de las disparidades de acumulación de renta capitalizada de suelo 1 y brecha de la renta (1UF=46US\$=100R\$)

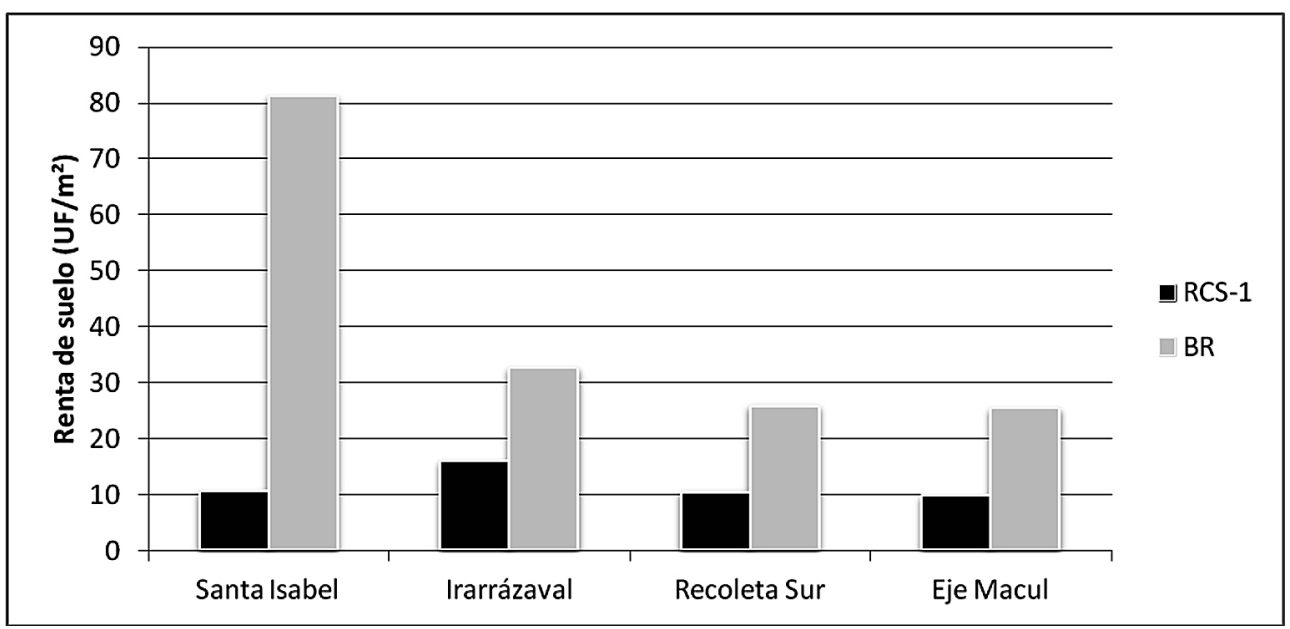

Fuente: Elaboración propia con información de la DOM de Santiago y portalinmobiliario.com. 
La zona que continúa después de Santa Isabel en orden decreciente de la brecha es Irarrázaval Poniente, aunque con una cifra mucho menor $\left(31,5 \mathrm{UF} / \mathrm{m}^{2}\right)$. Si bien esta zona presenta baja intensidad de producción de vivienda en sus proyectos, con edificaciones de altura media-baja y poca producción de departamentos por unidad de superficie de suelo desarrollado, su brecha levemente más alta a las dos zonas restantes se debe a que, como se mencionó en el párrafo anterior, presenta una oferta de departamentos más caros, con un promedio de 2.844 UF que incluso duplica precios medios de otras zonas. Por su parte, Recoleta $\left(29,9 \mathrm{UF} / \mathrm{m}^{2}\right)$ y Macul $\left(28,56 \mathrm{UF} / \mathrm{m}^{2}\right)$ presentan valores muy similares entre sí, siendo esta última la zona con menor brecha.

Interesa notar que, al revisar las tasas de acumulación, la situación varía en un aspecto importante. Es notorio que Santa Isabel posee tasas de acumulación muy superiores al resto de las zonas $(11,7)$, como es presumible por su alta brecha de renta, sucede que Irarrázaval $(2,2)$ aparece con tasas inferiores a Recoleta y Macul. Esto indica que la zona de Irarrázaval Poniente pasa a ser, entonces, una zona de alta brecha de renta pero con la tasa de acumulación más baja, lo que se debe a una participación muy alta de los propietarios originales de las zonas de renovación en el total de la renta obtenida por los agentes inmobiliarios. Como se señaló, Irarrázaval Poniente es la zona de mayor RCS-1, con valores que ascienden a $16 \mathrm{UF} / \mathrm{m}^{2}$, lo que reduce la tasa de acumulación de renta del desarrollo inmobiliario.

\section{Desplazamiento exclusionario}

Para examinar procesos de gentrificación, de la manera en que fue expuesto teórica y metodológicamente, es necesario observar la relación existente entre el valor recibido por hogares propietarios-residentes que venden sus predios a proyectos de renovación y los precios de las viviendas ofertadas para su relocalización residencial en el lugar. Con este propósito se realizó, para las cuatro zonas de estudio, un análisis de tasas de relocalización relativas a precios medios y mínimos ofertados en 2012.

A nivel general, se obtiene que el $59 \%$ de los propietarios-residentes encuestados obtiene tasas igual o mayor que 1 según precio medio, alcanzando con la renta obtenida de su propiedad a costear el departamento requerido en la misma zona de renovación. Del $41 \%$ de los casos que obtienen tasas menores a 1 , un $7,7 \%$ corresponde a propietarios que alcanzan a solventar menos de la mitad del precio del departamento que requieren; y un $25 \%$ solventa hasta el $80 \%$ del mismo precio (Figuras 6 y 7 ).

Al analizar la situación por zona se observan tasas de relocalización media considerablemente menores en Santa Isabel, donde un $17,5 \%$ de los propietarios-residentes encuestados (desde ahora la muestra) tienen tasas inferiores a 0.5 . Esto indica que la renta obtenida a obtener, de acuerdo a los valores medios registrados para la zona y la superficie predial de cada propietario, alcanza para costear menos de la mitad del precio del departamento que requiere el propietario 
Figura 6 - Tasas de relocalización residencial según precio medio en zonas de renovación urbana

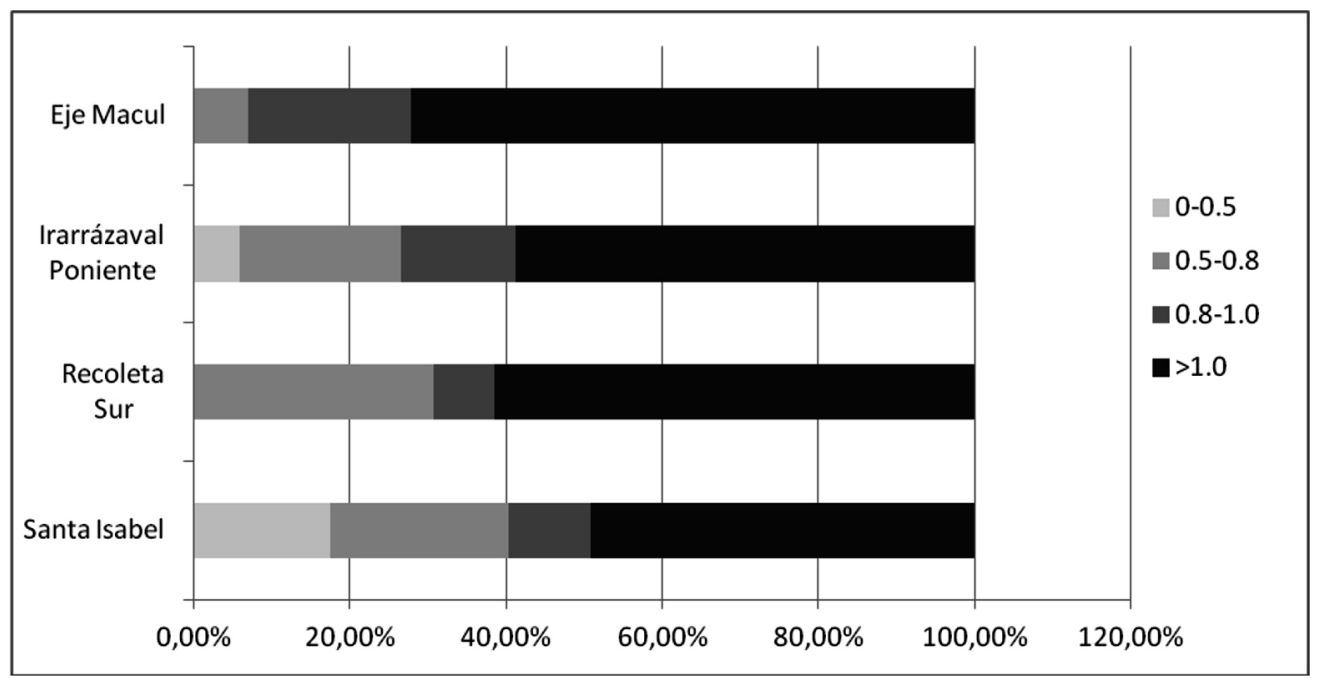

Fuente: Elaboración propia (2012).

Figura 7 - Modelo gráfico de arcos concéntricos de acceso a la vivienda. Se define por cada caso y su correspondiente tasa de relocalización

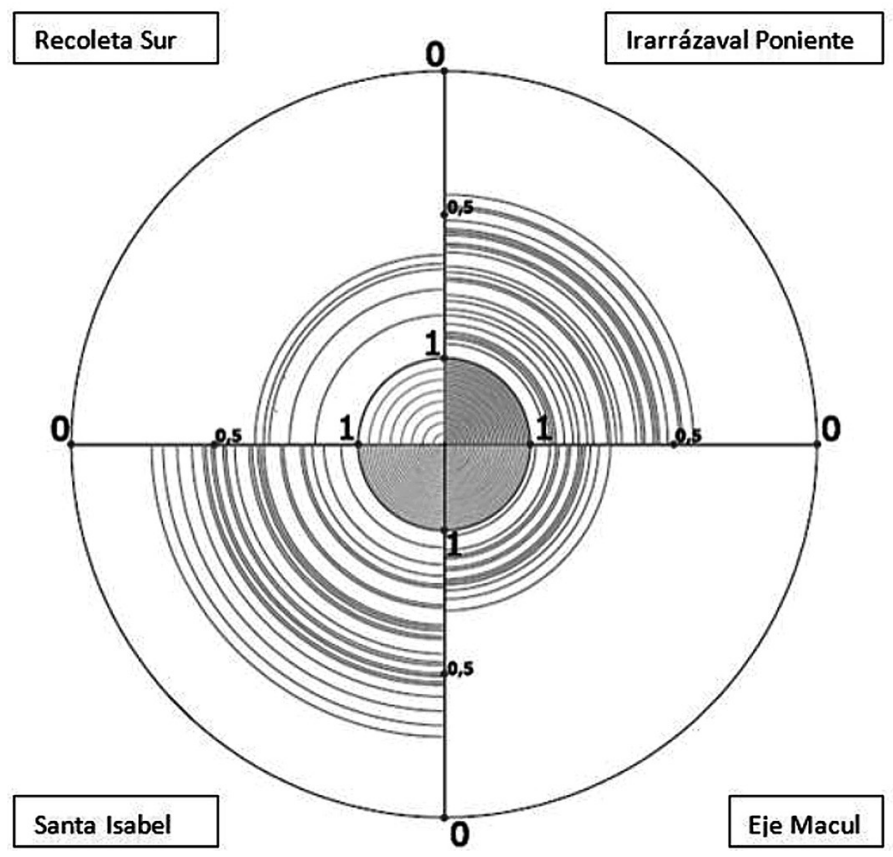

Fuente: Elaboración propia (2012). 
de acuerdo a la cantidad de habitantes de su hogar. Y solo un $49 \%$ de la muestra en la misma zona alcanzaría a solventar el precio del departamento requerido con la renta obtenida de su propiedad actual.

En contraposición, Macul aparece como la zona con mayor relocalización del peri-centro estudiado. No presenta tasas inferiores a 0.50 y solo un $7 \%$ se encuentra entre $0-5$ y 0.8 . De facto, un $72 \%$ de la muestra podría relocalizarse en esta misma zona, lo cual ya es una cifra considerablemente superior a la presentada en Santa Isabel. Por su parte, Irarrázaval Poniente sostiene un $6 \%$ de la muestra con tasas bajo los 0.5 y un total de $58 \%$ de residentes potencialmente relocalizados. El caso de Recoleta Sur, si bien considera una muestra mucho más pequeña (se encontraron menos propietarios-residentes, por ser una zona con alta presencia de arrendamiento), un $60 \%$ de los encuestados serían potencialmente relocalizados en la misma zona.

El factor explicativo de lo anterior es, fundamentalmente, la diferencia sustantiva en la superficie de las propiedades entre las zonas. Si bien en términos relativos el precio unitario del suelo $\left(U F / m^{2}\right)$ es similar, la superficie de las propiedades produce diferencias en la renta obtenida que determinan económicamente la relocalización de los residentes en las mismas zonas de renovación. Esta es la principal diferencia entre las zonas de Macul y Santa Isabel, aun cuando los precios de los departamentos sean levemente superiores en la primera. Lo relevante de esto es que al analizar las rentas de suelo registradas en el CBRS se dio cuenta de que éstas no sostenían relaciones indirectamente proporcionales con la superficie del predio, sino que mantenían valores constantes donde la superficie no es factor explicativo o determinante en el precio de la propiedad. Esto indica que es poco verosímil la consideración de que los predios más pequeños se puedan vender a mayor precio unitario de suelo, lo que permite prever escenarios más desfavorables para la relocalización en Santa Isabel.

Estas estimaciones están referenciadas al precio medio de los departamentos en cada zona. Al estimar las tasas de relocalización según el departamento de menor precio ofertado en el año 2012 (tasa de relocalización mínima), se obtiene que, a nivel general en el pericentro, el $81 \%$ de los encuestados tiene capacidad económica para relocalizarse en la zona de renovación respectiva de acuerdo a la renta obtenida. Del 19\% que no alcanza dicha situación, solo un $1 \%$ costea menos de la mitad del departamento. Si bien las cifras disminuyen previsiblemente al tratarse del departamento más barato ofertado en cada zona, sigue siendo importante una cifra cerca al $20 \%$ considerando que se trata de propietariosresidentes que no pueden acceder incluso a las viviendas de menor estándar producidas en el mercado inmobiliario de renovación.

Observando las tasas de relocalización mínima por zona, se obtiene que un 34\% de propietarios-residentes en Santa Isabel no tendrían capacidad de solventar el departamento más barato del mercado con la renta de su propiedad. Mientras, en Eje Macul la totalidad de los encuestados obtendría rentas superiores al precio del departamento más barato producido en dicha zona. Esta es una diferencia importante que encuentra situaciones intermedias en Irarrázaval Poniente y Recoleta Sur. En ambas, más de un $80 \%$ de 
los encuestados puede relocalizarse en el departamento de menor precio ofertado en la zona en el año 2012, lo cual ya es ampliamente superior a lo señalado para Santa Isabel.

\section{Conclusiones}

La gentrificación por renovación urbana en Santiago de Chile puede ser comprendida como un proceso de desplazamiento socioresidencial, específicamente de propietarios-residentes sin capacidad de relocalización in situ, producido en contextos de captura desigual de renta de suelo. A este respecto, el $41 \%$ de los hogares encuestados en las cuatro zonas no alcanza a solventar la vivienda que requiere para su relocalización in situ, lo cual es un indicador elocuente de la generalidad del proceso de gentrificación en el centro y pericentro metropolitano sujeto a renovación urbana residencial. $Y$ esto se desarrolla en un contexto en el que, mientras las utilidades inmobiliarias por unidad de suelo desarrollado crecen sostenidamente en el período de 2000 a 2012, con un aumento de un 104\%, los propietarios del suelo que residen en las zonas de renovación y que venden sus propiedades a las empresas inmobiliarias reciben rentas bastante constantes, que no varían más de un $5 \%$.

Comparando las distintas zonas estudiadas, se observa que Irarrázaval es la zona en donde los propietarios del suelo capturan una mayor proporción de la renta que genera el re-desarrollo inmobiliario. Por su parte, en Santa Isabel los desarrolladores inmobiliarios obtienen brechas de renta sustantivamente mayores, producto de la mayor cantidad de mercancías-vivienda que pueden producir por unidad de suelo; mientras los propietarios del suelo logran capturan rentas menores que en Irarrázaval, y similares a Recoleta y Macul. Sin embargo, considerando que en Santa Isabel los predios son bastante más pequeños que en estas otras dos zonas, con una cantidad importante bajo los $300 \mathrm{~m}^{2}$, es previsible que la relocalización de los propietarios-residentes sea más difícil. Al menos eso indican las tasas de relocalización en esta zona, donde tan solo un $49 \%$ de los hogares encuestados alcanzaría a solventar el precio del departamento requerido con la renta obtenida de su propiedad actual.

Las dos estimaciones realizadas en este estudio, relativas a la captura desigual de la renta del suelo y la relocalización de los propietarios-residentes, son una forma general de acercarse al problema de la gentrificación en Santiago de Chile. Considerando que la renovación urbana residencial en altura en el área central y pericentral es el proceso de mayor inversión y relevancia a nivel nacional, se torna imprescindible continuar con estudios de este tipo, que permitan problematizar y orientar una interpretación crítica desde la economía política del espacio urbano, atendiendo con principal interés los efectos de desplazamiento y exclusión que pueden afectar a las comunidades residenciales originarias.

De cierre, recalcar que el presente artículo pretende retomar los aportes de Neil Smith a la comprensión y análisis crítico del proceso de gentrificación desde un enfoque estructural marxista, ensayando adaptaciones metodológicas para aplicar la investigación en la realidad de Santiago 
de Chile. Dadas algunas similitudes en la estructuración urbana de las principales ciudades latinoamericanas, se espera haber aportado con un trabajo de alcance regional, que tenga perspectivas de ser replicado 0 re-situado en otras ciudades del continente que estén experimentando procesos de renovación urbana residencial en sus áreas centrales, bajo lógicas neoliberales de producción espacial.

\section{Ernesto López-Morales}

Universidad de Chile, Departamento de Urbanismo. Santiago, Región Metropolitana, Chile. elopez@uchilefau.cl

\section{Ivo Gasic Klet}

Universidad de Chile, Departamento de Urbanismo. Santiago, Región Metropolitana, Chile. igasic@uchilefau.cl

\section{Daniel Meza Corvalán}

Universidad de Chile, Departamento de Urbanismo. Santiago, Región Metropolitana, Chile. dmeza@uchilefau.cl

\section{Notas}

(1) Impulsado en las ciudades del norte global durante la desindustrialización de la década de 1980; ver Harvey (1989); Hamnett y Whitelegg, (2007).

(2) Más especificaciones sobre esta construcción teórica y sus posibilidades para estudios de gentrificación revísese López-Morales (2008, 2010a, 2010b, 2013).

(3) Una clase social que podría denominarse pequeño-burguesa por el carácter privado de sus emprendimientos, con pequeña inversión, y con un afán de distanciamiento social de las residencias tradicionales de los grupos sociales más altos. Esta clase compuesta por yuppies, artistas, bohemios, etc., invierten a pequeña escala sobre un barrio, lo que se traduce posteriormente en una apropiación material de los rentistas del mismo barrio para aumentar las rentas de alquiler o vender sus propiedades.

(4) Véase publicaciones de Schlack y Turnbull (2011) e Inzulza (2012).

(5) Primera Encuesta Metropolitana a residentes de barrios en renovación urbana en el Gran Santiago: Hacia una medición integral de efectos socio-espaciales de la renovación y el Mercado Inmobiliario. http://radio.uchile.cl/wp-content/uploads/2013/09/Primera-EncuestaMetropolitana Urbana.pdf o López et al., 2008. 
(6) En la encuesta mencionada se tomó el caso de 245 propietarios residentes (se excluyó de este análisis a los arrendatarios), a los cuales se les preguntó por la cantidad de habitantes de su hogar y se infirió la tipología de departamento requerida, siguiendo una lógica de 1 habitante = 1 dormitorio, 2 habitantes $=2$ dormitorios, 3-4 habitantes $=3$ dormitorios.

(7) Y, con ello, las rentas obtenidas por sus propietarios, toda vez que éstos estén ofertando localización y no las edificaciones internas del predio, como es el caso de este estudio.

\section{Referencias}

BORSDORF, A. e HIDALGO, R. (2013). Revitalization and tugurization in the historical centre of Santiago de Chile. Cities, v. 31, pp. 96-104.

CATTANEO, R. (2011). Los fondos de inversión inmobiliaria y la producción privada de vivienda en Santiago de Chile ¿Un nuevo paso hacia la financiarización de la ciudad? Eure, v. 37, n. 112, pp. 5-22.

CLARK, E. (1987). The rent gap and urban change: case studies in Malmö 1860-1985. Lund, Lund University Press.

DELGADILLO, V. (2008). Mejoramiento habitacional en las áreas urbanas centrales de América Latina. Del combate de tugurios a la rehabilitación habitacional progresiva. Revista INVI. Santiago, v. 23, n. 63, pp. 89-119.

DE MATTOS, C. (2006). Modernización capitalista y transformación metropolitana en América Latina: cinco tendencias constitutivas. In: GERAIGES DE LEMOS, A., ARROYO, M. e SILVEIRA, M. L. (eds.), Modernización capitalista y transformación metropolitana en América Latina: cinco tendencias constitutivas. São Paulo, Consejo Latinoamericano de Ciencias Sociales.

(2007). Globalización, negocios inmobiliarios y transformación urbana. Nueva Sociedad, n. 212, pp. 82-96.

GAJST, N. (2010) La escuela francesa de la regulación: Una revisión crítica. Vis. Futuro, v. 13, n.1. Disponível em: <http://www.scielo.org.ar/scielo.php?script=sci_arttext\&pid=S1668$87082010000100001 \&$ Ing=es\&nrm=iso>. ISSN 1668-8708. Acesso em: 7 jan 2013

HACKWORTH, J. (2007), The neoliberal city: governance, ideology, and development in American Urbanism. Ithaca, Cornell University Press.

HAMNETT, C. e WHITELEGG, D. (2007). Loft conversion and gentrification in London: from industrial to postindustrial land use. Environment and Planning A, v. 39, n. 1, pp. 106-124.

HARVEY, D. (1989). From Managerialism to entrepreneurialism: the transformation in urban governance in late capitalism. GeografiskaAnnaler. Series B, Human Geography, v. 71, n. 1, pp. 3-17,

HERZER, H. (2008). Con el corazón mirando al sur. Transformaciones en el sur de la ciudad de Buenos Aires. Buenos Aires, Espacio Editorial.

INZULZA-CONTARDO, J. (2012). Latino-Gentrification? Focusing on physical and socioeconomic patterns of change in latin american inner cities. Urban Studies. Illinois, n. 10, 2085-2107. 
JANOSCHKA, M. e CASGRAIN, A. (2011). Urbanismo neoliberal y gentrificación en Santiago de Chile: diálogos entre teoría urbana y reivindicación vecinal. Corporación Educacional Poblar. Santiago de Chile. Disponível em: http://penalolenvotano.bligoo.cl/media/users/17/863351/files/167929/ MPL_Gentrificacion.pdf. Acesso em: 2 fev 2013.

JANOSCHKA, M., SEQUERA, J. e SALINAS, L. (2013). Gentrification in Spain and LatinAmerica - a critical dialogue. International Journal of Urban and Regional Research, v. 38, n. 4, pp. 1234-1265.

LEES, L., SLATER, T. e WYLY, E. K. (2008). Gentrification. Londres, Routledge.

LÓPEZ-MORALES, E. (2008). Destrucción creativa y explotación de brecha de renta: discutiendo la renovaciónurbana del peri-centro sur poniente de Santiago de Chile entre 1990 y 2005. [En línea] Scripta Nova, v. 12, n. 270. Disponível em: http://www.ub.edu/geocrit/sn/sn-270/sn-270-100. htm. Acesso em: 30 jul 2012.

(2010a). Gentrification by ground rent dispossession: the shadows cast by large-scale urban renewal in Santiago de Chile. International Journal of Urban and Regional Research, v. 35, n. 2, pp. 330-357.

(2010b). Real estate market, state-entrepreneurialism and urban policy in the gentrification by ground rent dispossession of Santiago de Chile. Journal of Latin American Geography, v. 9, n. 1, pp. 145-173.

(2011). Gentrification by ground rent dispossession: the shadows cast by large scale urban renewal in Santiago de Chile. International Journal of Urban and Regional Research, v. 35, n. 2, pp. 1-28.

(2013). Urbanismo proempresarial y destrucción creativa: un estudio de caso de la estrategia de renovación urbana en el pericentro de Santiago de Chile, 1990-2005. Mexico, Infonavit-Redalyc.

LÓPEZ-MORALES, E.; GASIC, I. e MEZA, D. (2012). Urbanismo pro-empresarial en Chile: políticas y planificación de la producción residencial en altura en el pericentro del Gran Santiago. Revista INVI, v. 28, n. 76, pp. 75-114.

LÓPEZ-MORALES, E.; ARRIAGADA C., MEZA, D. e GASIC, I. (2013). Primera encuesta metropolitana a residentes de barrios en renovación urbana en el Gran Santiago: hacia una medición integral de efectos socio-espaciales de la renovación y el mercado inmobiliario. SEMINARIO FAU, Facultad de Arquitectura y Urbanismo, Universidad de Chile, 9 de septiembre. Disponível em: http://www. fau.uchile.cl/u/download.jsp?document=94934\&property=attachment\&index=0\&content=\&no cch $=20130926174204.0$

MARCUSE, P. (1985). Gentrification, abandonment and displacement: connections, causes and policy responses in New York City. Journal of Urban and Contemporary Law, v. 28, pp. 195-240.

ROJAS, E. (2004). Volver al centro. La recuperación de áreas urbanas centrales. Washington D.C., Banco Interamericano del Desarrollo.

SABATINI, F. (1990). Precios del suelo y edificación de viviendas (4 conclusiones sobre Santiago relevantes para políticas urbanas). Eure. Santiago, v. 16, n. 49, pp. 63-72.

(2000). Reforma de los mercados de suelo en Santiago, Chile: efectos sobre precios de la tierra y la segregación residencial. Eure. Santiago, v. 26, n. 77, pp. 49-80.

SCHLACK, E. e TURNBULL, N. (2011). Capitalizando lugares auténticos: artistas y emprendimientos en la regeneración urbana. $A R Q$. Santiago, n. 79, pp. 28-42. 
SHAW, K. (2008). A response to 'The Eviction of Critical Perspectives from Gentrification Research'. International Journal of Urban and Regional Research, v. 32, n. 1, pp. 192-194.

SLATER, T. (2006). The eviction of critical perspectives from gentrification research. International Journal of Urban and Regional Research, v. 30, n. 4, pp. 737-757.

(2009). Missing Marcuse: On gentrification and displacement. City - Analysis of Urban Trends Culture Theory Policy and Action, v. 13, n. 2, pp. 292-312.

SMITH, N. (1979). Toward a theory of gentrification a back to the city movement by capital, not people. Journal of the American Planning Association, v. 45, n. 4, pp. 538-548.

(1996). The new urban frontier: gentrification and the revanchist city. Londres/Nova York, Routledge.

(2002). New globalism, new urbanism: gentrification as global urban strategy. Antipode, v. XXXIV, n. 3, pp. 427-450.

VICUÑA, M. (2013). El marco regulatorio en el contexto de la gestión empresarialista y la mercantilización del desarrollo urbano del Gran Santiago, Chile. Revista Invi, v. 28, n. 78. Disponível em: http:// revistainvi.uchile.cl/index.php/INVI/article/view/761/1100. Acesso em: 2 out 2013.

WEBER, R. (2002). Extracting value from the city: neoliberalism and urban redevelopment. Antipode, v. XXXIV, n. 3, pp. 519-540.

Texto recebido em 16/out/2013

Texto aprovado em 19/fev/2014 